\title{
Multicenter Epidemiological Study of Osteoporotic Fractures in Eurasia (EVA Study). A step towards reducing the burden of age- related diseases
}

\author{
Alla Zakroyeva ${ }^{1, *}$, Olga Lesnyak ${ }^{2}$, Sarkis Sahakyan ${ }^{3}$, Georiy Ramanau ${ }^{4}$, Viktor Kazak ${ }^{5}$, \\ Samal Issayeva ${ }^{6}$, and Munavvar Shakirova ${ }^{7}$ \\ ${ }^{1}$ Ural State Medical University, 620028, Repina Str., 3, Ekaterinburg, Russia \\ ${ }^{2}$ Mechnikov North West State Medical University, 191015, Kirochnaya Str., 41, St.Petersburg, Russia \\ ${ }^{3}$ Artashat Medical Center, 0701, Aram Khachaturyan Str., 7, Artashat, Armenia \\ ${ }^{4}$ Gomel State Medical University, 246050, Lange Str., 5, Gomel, Belarus \\ ${ }^{5}$ State University of Medicine and Pharmacy, 2004, Stefan cel Mare si Sfant blvd., 165, Chisinau, \\ Republic of Moldova \\ ${ }^{6}$ Asfendiyarov National Medical University, 050000, Tole bi Str., 94, Almaty, Kazakhstan \\ ${ }^{7}$ Republican Medical Center for Endocrinology, Tashkent, Uzbekistan
}

\begin{abstract}
To extend elderly patients' active longevity, practitioners all over the world need a precise clinical instrument to assess the 10-year osteoporotic fractures probability and choose the optimal preventive intervention. The purpose of this research is to assess major osteoporotic (OP) fractures incidence in CIS countries and to create country-specific computing FRAX clinical prediction tools. Method. We carried out a cohort population-based study (EVA) in Russia, Belarus, Armenia, Moldova, Kazakhstan, and Uzbekistan in 2011-2018. The age- and sexspecific OP fracture incidence values were incorporated into the computing FRAX models. Results. We revealed that the highest standardized HP incidence was obtained in Kazakhstan (338 and 255 per 100000 per year for women and men older than 50 years resp.) and Moldova (331,0 and 155,0 per year for women and men resp.), the lowest in Armenia (201 and 136 per 100000 per year for women and men resp.). The FRAX models showed that in Kazakhstan, Uzbekistan, and Moldova, elderly people had the highest hip fracture probabilities. Estimates reveal that the OP fractures numbers in the EVA-project countries will grow by $1.5-3$ times by 2050 . Conclusion. The created national FRAX instruments should enhance the accuracy of determining fracture probability among older people, help clinicians to make decisions concerning OP prophylactic, and to step forward to reducing the burden of age-related diseases.
\end{abstract}

\section{Introduction}

\footnotetext{
*Corresponding author: zakroeva.alla@ mail.ru
} 
With the population aging, the prevalence of chronic age-related diseases such as osteoporosis and fragility fractures caused thereby is increasing considerably. In Europe the annual cost of fractures associated with osteoporosis exceeded $€ 37$ billion in 2010 [1], and disability due to osteoporosis was greater than that caused by any single cancer, with the exception of lung cancer [2]. Fortunately, a wide variety of treatments are available that favourably affect bone mass and therefore decrease the risk of fractures associated with osteoporosis [3]. The use of such interventions by health care practitioners is assisted by instruments that assess patients' fracture risk to optimize clinical decisions concerning prevention and treatment. The most widely used web-based tool FRAX® (https://www.sheffield.ac.uk/FRAX/) meets these requirements and computes the 10-year probability of fragility fractures based on several common clinical risk factors and, optionally, on a DXA scan result $[4,5]$. However, reliable epidemiologic characteristics of osteoporosis that would allow researchers to evaluate its burden and to create countryspecific FRAX instruments are absent in most of the former USSR region.

The purpose of this research is to assess major osteoporotic (OP) fractures incidence in CIS countries and to create country-specific FRAX clinical prediction tools for them. FRAX instruments will allow practitioners to assess 10-year osteoporotic fractures probability in elderly patients and choose the optimal intervention, decreasing fragile fracture risk as well as extending patients' active longevity.

\section{Method}

We conducted a population-based multicentred study (EVA) in Russia, Belarus, Armenia, Moldova, Kazakhstan, and Uzbekistan in 2011-2018. At the first stage, in 2011-2012, data concerning proximal hip fractures (HP), distal forearm, and proximal humerus fractures in people older than 40 years were gathered retrospectively. During the subsequent 2-years prospective study, we collected data on patients with OP fractures from all reliable sources including primary care physicians. The industrial cities or big rural regions of EVA countries that had geographic, administrative, and medical conditions as well as demographic parameters close to the whole country were selected as the objects of investigation.

Case report forms were developed to record the patient's age, sex, and place of residence as well as date, character of injury, and ICD -10 code. In addition, the official medical records of hip fractures in men and women from the central cities' hospital registers, the outpatient trauma unit's data, GP data, and all emergency service data as well as coroner cases were reviewed. We also engaged home-visiting nurses who were instructed to notify traumatologists about each suspected case that had not been referred to hospital attention. An orthopaedic surgeon subsequently examined all such cases. The diagnosis was verified clinically or, where possible, by X-ray examination. Additionally, in Armenia and Uzbekistan, we contacted folk healers and the elders of the administrative communities. They identified housebound individuals who were subsequently visited at home by the district nurse and redirected all suspected patients to the Central hospital trauma centers of the regions.

The reason for accessing multiple sources of information including that from primary care was to identify patients with hip fractures who were not admitted to hospital obtaining in pilot study Russia. There, like in other former USSR countries of Eastern Europe, facilities for surgical management are limited so that hospital admission was not often feasible.

To avoid double counting, further admissions for the same fracture site in the observation time were excluded. In some documents, the fracture ICD-10 code was not specified. In such cases, radiographs were retrieved, and fractures, if verified, were 
included into the database. Permanent residence in the region under research was a criterion for inclusion. High energy fractures were excluded (falls from heights exceeding a standing height). We excluded pathological fractures attributable to cancer with metastases or to multiple myeloma.

Age- and sex-specific incidence in each country was applied to the population in 2015 to estimate the number of hip fractures nationwide. Additionally, future projections were estimated up to 2050 assuming that age- and sex-specific incidence remained stable. Population demography was taken from the United Nations using the medium variant for fertility [6].

Data on osteoporotic fracture rate were used to construct country-specified FRAX models. The development and validation of FRAX have been extensively described $[4,5]$. The risk factors used were based on a systematic set of meta-analyses of population-based cohorts worldwide and validated in independent cohorts with over 1 million patient-years of follow-up. The construct of the FRAX models for EVA countries retained the beta coefficients of the risk factors in the original FRAX model with the incidence rates of hip fracture and mortality rates for Russia, Belarus, Armenia, Moldova, and Kazakhstan. National mortality rates used data from the World Health Organization for 2014 [7]. Tenyear fracture probabilities were compared to the countries where FRAX models were available.

\section{Results}

OP fractures incidence varied substantially even in neighboring geographic areas, but in all countries, the substantial part of HP patients did not undergo hospital treatment. In Uzbekistan this proportion was the maximum and reached $66 \%$. High rates of nonadmittance patients have also been reported in Pervouralsk, Russia (27\%), Armenia (44\%), and Belarus (29\% of cases) and Kazakhstan (29\%) [8-11]. Moreover, the number of people that did not underwent specialized traumatological care increased dramatically with age, especially in Uzbekistan. These hidden hip fracture cases that escaped from official medical statistics reinforce a view that data on hip fracture based solely on hospital records are unreliable in this region of the world.

The highest standardized HP incidence was obtained in Kazakhstan (338 and 255 per 100000 per year for women and men older 50 years resp.) and Moldova (331,0 and 155,0 per year for women and men resp) [11-13], the lowest rate in Armenia (201 and 136 per 100000 per year for women and men resp.). As expected, women in all countries were more likely to have OP hip fractures (crude female/male ratio was 1,2-1,7). Above the age of 70 years, hip fracture rate difference among female and male amounted to $3,1-4,1$. The estimated annual OP proximal femur fracture incidence increased with age in both sexes exponentially up to the age of 90, though more markedly in women. Incidence in women in Moldova, Kazakhstan, and Uzbekistan rose most steeply and reached 4199, 4167, and 3571 $/ 100000$ per year in the oldest. This distribution is showed in Fig.1. 


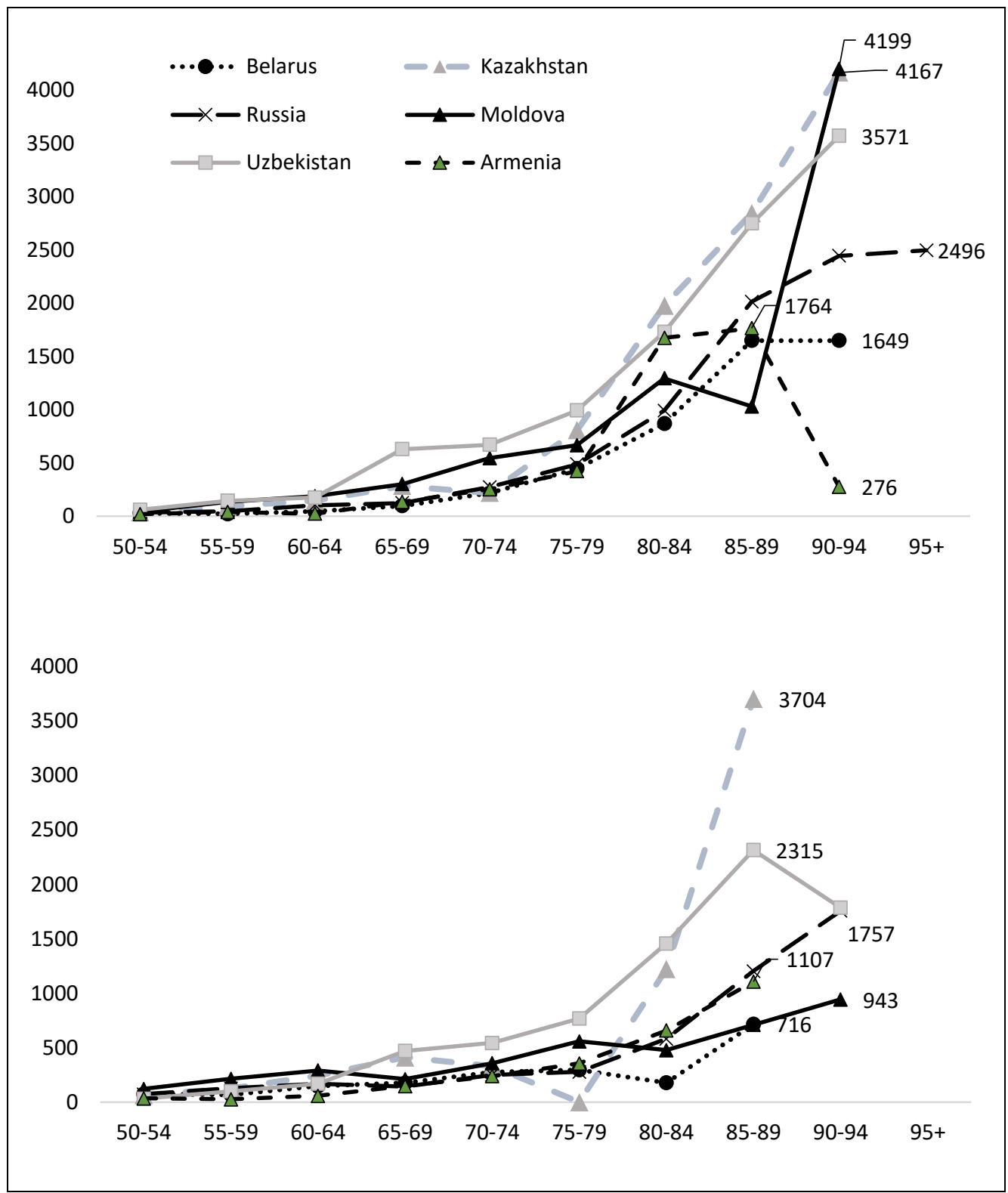

Fig. 1. Annual incidence of osteoporotic hip fractures among women (upper panel) and men (lower panel) in the EVA-project countries in various age groups per 100000

The discovered epidemiologic data were incorporated into the models for the computer FRAX construction for all countries using J. Kanis's method. We took into account the fact that in countries that calculated proximal forearm and humerus fracture incidence in addition to the HF rate, the number of upper extremity fractures was much greater than would be expected based on a similar epidemiologic ratio in Western Europe. These peculiarities allowed us to create more precise specific prognostic FRAX tools for the EVA project countries.

The FRAX model predicts that the life-time hip fracture risk above the age of 50 years in the Kazakh population (12,6\% and $6 \%$ for women and men resp.,), Uzbekistan (14,7\% 
and $8,7 \%$ for women and men resp..), and Moldova (9,3\% and 5,7\% for women and men resp.) are close to each other but differ substantially from China projections $(5,9 \%$ and 3,3\% resp). Kazakhstan, Uzbekistan, and Moldova were therefore categorized as countries with "high" probabilities of fragile fractures for older people, other were characterized as showing a "moderate" risk. Among the EVA countries, life-time probability of hip fracture above the age of 50 was maximal and minimal in Russia $(7,7 \%$ for women and 3,8\% for men).

Assuming that the regional OP fracture rate ranks were representative for all countries we calculated, based on the UN demographic estimates for 2015, the annual number of OP fractures in men and women aged 50 and older in all survey countries in 2015. Furthermore, we found that according to the UN demographic prognostic of population aging, the nationwide OP fractures numbers in the EVA-project countries will grow by 1.53 times by 2050. The highest growth of the OP burden is predicted in Kazakhstan, where the proportion of people older than 50 is about to increase by $89 \%$ by 2050 compared with 2015.

\section{Conclusion}

For the first time, national FRAX instruments have been created for the Republics of the CIS based on accurate regional population-based OP fracture estimates. These FRAX models should enhance the accuracy of determining fracture probability among older people, help clinicians to guide decisions about OP prophylactic, and move forward to reducing the burden of age-related diseases.

\section{References}

1. E. Hernlund, A. Svedbom, M. Ivergård, J. Compston, C. Cooper, J. Stenmark, E.V. McCloskey, B. Jönsson, J.A. Kanis, Archives of Osteoporosis, 8, 136 (2013)

2. O. Johnell, J.A. Kanis, Osteoporos Int., 17, 1726-33(2006)

3. J.A. Kanis, C. Cooper, R. Rizzoli, J-Y. Reginster, Osteoporos Int., 30, 3-44 (2019)

4. J.A. Kanis on behalf of the World Health Organization Scientific Group Assessment of osteoporosis at the primary healthcare level. Technical Report (2008). WHO Collaborating Centre, University of Sheffield, UK. URL: http://www.shef.ac.uk/FRAX/pdfs/WHO_Technical_Report.pdf.

5. J.A. Kanis, O. Johnell, A. Oden, H. Johansson, E. McCloskey, Osteoporos Int., 19, 385-397 (2008)

6. United Nations Department of Economic and Social Affairs, Population Division. World Population Prospects (2017) URL: https://www.worldometers.info/worldpopulation/

7. World Health Organization Health statistics and information systems (2018) URL: http://www.who.int/healthinfo/statistics/mortality_rawdata/en/

8. O. Lesyak, O. Ershova, K. Belova, E. Gladkova, O. Sinitsina, O. Ganert, M. Romanova, V. Khodirev, H. Johansson, E. McCloskey, J.A. Kanis, Arch. Osteoporos., 7, 67-73 (2012)

9. H. Ramanau, I. Chernyanin, E. Rudenka, O. Lesnyak, A. Zakroyeva, JP. Bilezikian, H. Johansson, N.C. Harvey, E. McCloskey, J.A. Kanis, Arch. Osteoporos., 13(1), 42 doi: 10.1007/s11657-018-0454-4 (2018).

10. O. Lesnyak, S. Sahakyan, A. Zakroyeva, JP. Bilezikian, N. Hutchings, V. Babalyan, R. Galstyan, A. Lebedev, H. Johansson, NC. Harvey, E. McCloskey, J.A. Kanis, Arch. Osteoporos., 12(1), 98 (2017) doi: 10.1007/s11657-017-0392-6 
11. A. Zakroyeva, O. Lesnyak, V. Cazac, L. Groppa, E. Russu, L. Chislari, L. Rotaru, H. Johansson, N.C. Harvey, E. McCloskey, M. Lorentzon, J.A. Kanis, Arch. Osteoporos., 15(1), 13 (2020)

12. S. Issayeva, O. Lesnyak, A. Zakroyeva, B. Issayeva, D. Dilmanova, H. Johansson, E. Liu, M. Lorentzon, N.C. Harvey, E. McCloskey, J.A. Kanis, Arch. Osteoporos., 15(1), 30 (2020) 